\title{
Coupling Scale-Free and Classical Random Graphs
}

\author{
Béla Bollobás and Oliver Riordan
}

Abstract. Recently many new "scale-free" random graph models have been introduced, motivated by the power-law degree sequences observed in many large-scale real-world networks. The most studied of these is the Barabási-Albert growth with "preferential attachment" model, made precise as the LCD model by the present authors. Here we use coupling techniques to show that in certain ways the LCD model is not too far from a standard random graph; in particular, the fractions of vertices that must be retained under an optimal attack in order to keep a giant component are within a constant factor for the scale-free and classical models.

\section{Introduction}

Recently many variants of the following question have been considered: how vulnerable are scale-free graphs to attack? To form a specific question we must choose a suitable graph model, and a notion of vulnerability. We start with the model.

The most studied, and perhaps the most basic scale-free graphs are those introduced in vague terms by Barabási and Albert in [Barabási and Albert 99]. They, and many, but not all, authors use "scale-free" to mean having power-law distribution of, for example, the degree sequence - a feature observed in many real-world graphs. See, for example, the extensive surveys [Albert and Barabási 02, Dorogovtsev and Mendes 02] for general background. In any case, whatever the terminology, the key features of the Barabási-Albert model are that the graph grows one vertex at a time, each new vertex sending edges to randomly chosen

(C) A K Peters, Ltd.

I542-795I/04 $\$ 0.50$ per page 
old vertices, selected with probabilities proportional to their degrees; this leads to a power-law distribution for the degree sequence.

The original definition of the Barabási-Albert model in [Barabási and Albert 99] (repeated in the survey [Albert and Barabási 02]) is not properly specified in the growth step, and there is a problem getting started. See [Bollobás and Riordan 04] for the details, or [Bollobás and Riordan 02] for a fuller discussion, as well as a partial survey of rigorous mathematical results in this area. To prove mathematical results, something definite and precise is needed, and this is one reason for the introduction of the "Linearized Chord Diagram" or LCD model in [Bollobás and Riordan 04]. The other reason is that this particular model has a special property that makes it convenient for mathematical work, namely a simple static description, via random pairings or LCDs (see [Bollobás and Riordan 04]). Here we shall not need this static description, working instead with the dynamic one.

As for the Barabási-Albert model, the LCD model $G_{m}^{(n)}$ of [Bollobás and Riordan 04] is determined by an integer parameter $m \geq 1$, giving the number of edges per vertex. The $n$-vertex LCD graph $G_{m}^{(n)}$ is constructed one vertex at a time, starting from an empty "graph" with no vertices (or, if one prefers, a single vertex with $m$ loops, or any other fixed given graph). At each step, a new vertex is added. Then $m$ edges from this new vertex are added one by one; the other end of each edge is a vertex chosen randomly, with probability proportional to degree (more precisely, to avoid problems with zero degrees, we use degree at the time, counting the previously added edges, and the "first half" of the edge being added). A more formal definition is given in the next section.

The robustness under random failure and vulnerability to attack of the BarabásiAlbert and LCD models have been considered in several papers, including [Albert et al. 00, Bollobás and Riordan 03, Callaway et al. 00, Cohen et al. 00, Cohen et al. 01]. In all but [Bollobás and Riordan 03], the results are either experimental, or heuristic. Roughly speaking, it turns out that $G_{m}^{(n)}$ is much more robust to random failures than classical random graphs with the same number of vertices and edges, but also more vulnerable to attack. When analyzing vulnerability, there are various kinds of attack one could consider, and various measures of its success. In all cases, we measure the success of the attack by looking at the size of the largest component in the graph that remains after deleting a certain number of vertices. In [Bollobás and Riordan 03], which is the only rigorous mathematical work listed above, the simplest possible attack on $G_{m}^{(n)}$ is considered: delete the oldest vertices, as they are the ones with the largest expected degrees. The simplicity of this attack makes precise analysis possible, but it is still far from easy. In experimental and heuristic work, other attacks are considered: either delete vertices starting with those of highest degree [Callaway et al. 
00], or delete the vertex of highest degree, then the vertex of highest degree in the graph that remains, and so on [Albert et al. 00]. There turns out to be little difference between these attacks.

Here we shall analyze rigorously the best possible attack: delete a given number of vertices so as to minimize the size of the largest component in what remains. Due to the complexity of this attack we can only give results within a constant factor. However, the method itself should be useful for studying other properties of scale-free graphs. Loosely speaking, we shall show that there are two classical random graphs whose sizes differ by a constant factor, such that $G_{m}^{(n)}$ contains almost all of the first, and is almost entirely contained in the second. The precise details of the results we prove are tuned for the application, but the method should be useful in other contexts.

Before getting started, let us note that everything we do here applies equally well to certain other models, in particular, to (a precise version of) the "preferential attachment with initial attractiveness" models of Dorogovtsev, Mendes, and Samukhin [Dorogovtsev et al. 00], and Drinea, Enachescu, and Mitzenmacher [Drinea et al. 01], whose degree distribution is analyzed rigorously in certain cases by Buckley and Osthus [Buckley and Osthus 04]. Such models give power-law degree distributions with variable exponents, rather than the fixed exponent given by the Barabási-Albert or LCD model.

\section{Definition of the Model}

In [Bollobás and Riordan 04], the LCD model, $G_{m}^{(n)}$, is defined as follows:

For precise definitions, we start with the case $m=1$. Consider a fixed sequence of vertices $v_{1}, v_{2}, \ldots$. (Most of the time we shall take $v_{i}=i$ to simplify the notation.) We write $d_{G}(v)$ for the degree of the vertex $v$ in the graph $G$. We shall inductively define a random graph process $\left(G_{1}^{t}\right)_{t \geq 0}$ so that $G_{1}^{t}$ is a graph on $\left\{v_{i}: 1 \leq i \leq t\right\}$, as follows. Start with $G_{1}^{0}$, the empty "graph" with no vertices, or with $G_{1}^{1}$ the graph with one vertex and one loop. Given $G_{1}^{t-1}$, we form $G_{1}^{t}$ by adding the vertex $v_{t}$ together with a single edge between $v_{t}$ and $v_{i}$, where $i$ is chosen randomly with

$$
\operatorname{Pr}(i=s)= \begin{cases}d_{G_{1}^{t-1}}\left(v_{s}\right) /(2 t-1) & 1 \leq s \leq t-1 \\ 1 /(2 t-1) & s=t\end{cases}
$$

In other words, we send an edge $e$ from $v_{t}$ to a random vertex $v_{i}$, where the probability that a vertex is chosen as $v_{i}$ is proportional to 
its degree at the time, counting $e$ as already contributing one to the degree of $v_{t} \ldots$ For $m>1$ we add $m$ edges from $v_{t}$ one at a time, counting the previous edges as well as the "outward half" of the edge being added as already contributing to the degrees. Equivalently, we define the process $\left(G_{m}^{t}\right)_{t \geq 0}$ by running the process $\left(G_{1}^{t}\right)$ on a sequence $v_{1}^{\prime}, v_{2}^{\prime}, \ldots$; the graph $G_{m}^{t}$ is formed from $G_{1}^{m t}$ by identifying the vertices $v_{1}^{\prime}, v_{2}^{\prime}, \ldots, v_{m}^{\prime}$ to form $v_{1}$, identifying $v_{m+1}^{\prime}, v_{m+2}^{\prime}, \ldots, v_{2 m}^{\prime}$ to form $v_{2}$, and so on.

Note that this is just a more formal way of writing the description of $G_{m}^{(n)}$ given in the introduction. For the rest of the paper we take $v_{i}=i$, so $G_{m}^{(n)}$ is a graph on the vertex set $[n]=\{1,2, \ldots, n\}$.

The standard random graph model we shall use for comparison is $G(n, p)$, a graph on $[n]$ where each pair of vertices is joined independently with probability $p$. This is often known as the Erdős-Rényi random graph: while they proved many fundamental results about $G(n, p)$, their original model $G(n, M)$ [Erdős and Rényi 59] was slightly different. $G(n, p)$, which is much easier to work with, was actually introduced by Gilbert [Gilbert 59].

\section{Results}

Throughout, whp means with high probability, i.e., with probability tending to 1 as $n \rightarrow \infty$, with all other parameters (e.g., $m$ ) fixed.

Theorem 3.I. There are absolute constants $m_{0}$ and $c>0$ such that, for all $m \geq$ $m_{0}$, whp the graph $G_{m}^{(n)}$ has the following two properties. (a) Every induced subgraph of $G_{m}^{(n)}$ on at least $10 n \log m / m$ vertices contains a component of order at least $2 n \log m / m$. (b) The graph $G_{m}^{(n)}$ contains an independent set of order cn $\log m / m$.

Property (a) shows that whatever method we use to delete vertices from $G_{m}^{(n)}$, all but a fraction $\Theta(\log m / m)$ must be deleted to make the graph "fall apart," i.e., to avoid leaving a component of order $\Theta(n)$. For comparison, when deleting vertices from the start, all but a fraction $\Theta(1 / m)$ must be deleted. Property (b) shows that our result is best possible apart from the constant for general attacks. It remains an interesting question to obtain a result for the specific attack considered by Albert, Jeong, and Barabási [Albert et al. 00], although intuition from standard random graphs suggests that this attack will not work much better than a naïve attack, probably also requiring the deletion of all but a fraction $\Theta(1 / m)$ of the vertices, but with a different implicit constant. 
The proof of Theorem 3.1 is based on two coupling results comparing $G_{m}^{(n)}$ with a standard random graph $G(n, p)$ in which each pair of vertices is joined independently with probability $p$. In the statements below, $\sim$ means "has the same distribution as." Given two graphs $G_{1}, G_{2}$ on the same vertex set, we write $e\left(G_{1} \backslash G_{2}\right)$ for the number of edges present in $G_{1}$ but not in $G_{2}$.

Theorem 3.2. Let $\eta<1 / 2$ be fixed. Then there exist constants $A, c>0$, independent of $m$, such that for each fixed $m$ we may construct coupled random graphs $G_{1}$ and $G_{2}$ on the same vertex set, with $G_{1} \sim G_{m}^{(n)}$ and $G_{2} \sim G(n, \eta m / n)$, such that whp $e\left(G_{2} \backslash G_{1}\right) \leq A e^{-c m} n$.

In other words, if $m$ is large then we can couple $G_{m}^{(n)}$ and a standard random graph $G(n, p)$ with roughly a quarter as many edges in such a way that $G_{m}^{(n)}$ contains almost all edges of the standard random graph.

Theorem 3.3. Let $\epsilon>0$ be given. Then there is a constant $C$, independent of $m$, such that for each fixed $m$ we may construct coupled random graphs $G_{1}$ and $G_{2}$ on the same vertex set, with $G_{1} \sim G_{m}^{(n)}$ and $G_{2} \sim G(n, C m / n)$, such that whp $G_{2}$ contains $G_{1} \backslash V$ for some set $V$ of vertices with $|\{i \in V: i \geq \epsilon n\}| \leq \epsilon n / m$.

In other words, if $m$ is large then we can couple $G_{m}^{(n)}$ and a standard random graph $G(n, p)$ with a constant times as many edges in such a way that almost all of $G_{m}^{(n)}$ is contained in the standard random graph. Although this result is stated in terms of numbers of vertices, a corresponding result for numbers of edges follows immediately. In Theorem 3.3, we control not only the number of vertices that must be deleted, but also where they are.

Although the precise forms of Theorems 3.2 and 3.3 are chosen for the application to Theorem 3.1, they themselves, or similar results proved by the same method, are likely to be useful in other applications for showing that, in a certain sense, $G_{m}^{(n)}$ is not all that far from a standard random graph.

\section{Proofs}

We start with the proof of Theorem 3.2.

Proof of Theorem 3.2. For simplicity in the proof, we make no effort to optimize the constants. We construct the graphs $G_{1}$ and $G_{2}$ on the vertex set $[n]=$ $\{1,2, \ldots, n\}$ one vertex at a time. In fact, $G_{1}$ will be a multigraph (loops and multiple edges are allowed), constructed according to the inductive definition of 
$G_{m}^{(n)}$. Let $k_{0}$ be a slowly growing function of $n$ to be chosen later. We start by constructing graphs $G_{i}\left[k_{0}\right], i=1,2$, on $\left[k_{0}\right]$ with the appropriate distributions, making no assumptions about the coupling of these initial graphs.

Suppose that we have constructed $G_{1}[k]$ and $G_{2}[k]$, the subgraphs of $G_{1}$ and $G_{2}$ induced by the first $k$ vertices, with $G_{1}[k] \sim G_{m}^{(k)}$ and $G_{2}[k] \sim G(k, \eta m / n)$. We consider adding the $m$ edges of $G_{1}$ from vertex $k+1$ to earlier vertices, according to the preferential attachment rule (2.1). Let the $i$ th edge added join vertex $k+1$ to vertex $t_{i}, 1 \leq t_{i} \leq k+1$. Note that the sum of the vertex degrees in $G_{1}[k]$ is exactly $2 m k$, and that, during the addition of the $m$ edges from vertex $k+1$, the sum of the degrees of all $k+1$ vertices cannot exceed $2 m k+2 m$. Note also that each vertex of $G_{1}[k]$ has degree at least $m$. Hence, thinking of $G_{1}[k]$ as fixed, given $t_{1}, \ldots, t_{i-1}$, the probability for any $1 \leq j \leq k$ that $t_{i}=j$ is at least $m /(2 m k+2 m)=1 /(2 k+2)$. We may thus construct independent random variables $s_{i}, 1 \leq i \leq m$, taking values in $[k] \cup\{\emptyset\}$, such that for each $i$ and each $j, 1 \leq j \leq k$, we have $\operatorname{Pr}\left(s_{i}=j\right)=1 /(2 k+2)$, and so that if $s_{i} \neq \emptyset$ then $t_{i}=s_{i}$. (Here $s_{i}=\emptyset$ is a convenient way of writing " $s_{i}$ undefined.") To do this, we can consider a portion of the event $t_{i}=j$ with probability $1 /(2 k+2)$ as arising from uniform attachment, setting $s_{i}=j$ in this case, and the rest from some more strongly biased preferential attachment, setting $s_{i}=\emptyset$.

Let $X$ be the number of values $i$ for which $s_{i} \neq \emptyset$, noting that $X$ has a binomial $\operatorname{Bi}(m, k /(2 k+2))$ distribution. Note that $X$ is independent of $G_{1}[k]$. We shall construct a random variable $Y$ with a $\operatorname{Bi}(k, \eta m / n)$ distribution, which will be the degree of vertex $k+1$ in $G_{2}[k+1]$. Note that, as $\eta<1 / 2$ and $k \leq n$, while $k$ is large, we have $\mathbb{E}(X)=m k /(2 k+2) \geq(1+\epsilon) \eta m k / n=(1+\epsilon) \mathbb{E}(Y)$ for some constant $\epsilon>0$ depending on $\eta$ but not on $m$. It follows from standard inequalities (for example, the Chernoff bounds) that the probability that a binomial deviates from its mean by more than a constant factor is exponentially small in the mean. Thus, we may couple $X$ and $Y$ so that with probability at least $1-A e^{-c^{\prime} m}$ we have $Y \leq X$, for some $A, c^{\prime}>0$.

Now with probability $1-O\left(m^{2} / k\right)=1-o(1)$, the values other than $\emptyset$ taken by the $s_{i}$ are distinct, and, conditional on this event and on $X$, these values are a subset $S_{1}$ of $[k]$ of size $X$ distributed uniformly among all such subsets. Let us construct $G_{2}[k+1]$ form $G_{2}[k]$ by adding edges from $k+1$ to all vertices in a subset $S_{2}$ of $[k]$ with $\left|S_{2}\right|=Y$, chosen uniformly among all such subsets. Let us couple the uniform choices so that $S_{2}$ is contained in $S_{1}$ if $Y \leq X$. Let $D_{k+1}$ be the number of edges of $G_{2}[k+1]$ incident with vertex $k+1$ not contained in $G_{1}[k+1]$, so from the definitions $D_{k+1} \leq\left|S_{2} \backslash S_{1}\right|$. From the couplings of $X$ with $Y$ and of $S_{1}$ with $S_{2}$, by the remarks above we have

$$
\operatorname{Pr}\left(D_{k+1}>0\right)=A e^{-c^{\prime} m}+o(1) .
$$


Also, $D_{k+1}$ is highly unlikely to be very large. In particular, $D_{k+1}$ is bounded by $Y$, a binomial random variable with mean at most $m / 2$. Thus, from the Chernoff bounds, the probability that $D_{k+1}$ exceeds $m$ is exponentially small in $m$. Together with (4.1) this shows that for $n$ large enough we have

$$
\mathbb{E}\left(D_{k+1}\right) \leq A m e^{-c^{\prime} m}
$$

changing the constants $A, c^{\prime}$ if necessary.

Theorem 3.2 follows by observing that the total number of edges of $G_{2} \backslash G_{1}$ is bounded by $\left(\begin{array}{c}k_{0} \\ 2\end{array}\right)+\sum_{k=k_{0}+1}^{n} D_{k}$. The sum of the $D_{k}$ has expectation at most $A m n e^{-c^{\prime} m}$. Moreover, it is easy to check that this sum is concentrated around its expectation, using the independence of each step in the construction. Also, the term $\left(\begin{array}{c}k_{0} \\ 2\end{array}\right)$ is negligible provided we choose $k_{0}=k_{0}(n)$ to tend to infinity sufficiently slowly, so the result follows, replacing $c^{\prime}$ by a larger constant $c$ to absorb the initial factor of $m$.

The first claim in Theorem 3.1 follows by a straightforward calculation.

Proof of Theorem 3.I(a). We apply Theorem 3.2 with $\eta=6 / 13$. Let $m_{0}$ be a large constant to be chosen later, and let $m \geq m_{0}$ be fixed. Construct coupled graphs $G_{1}$ and $G_{2}$ as described in Theorem 3.2. Suppose that $V$ is a set of $10 n \log m / m$ vertices of $G_{1} \sim G_{m}^{(n)}$ inducing a subgraph with no component of order at least $2 n \log m / m$. (We ignoring rounding to integers, which makes no essential difference.) Then $V$ has a partition $V_{1}, V_{2}$ with $\left|V_{i}\right| \geq 4 n \log m / m$, $i=1,2$, such that $G_{1}$ contains no $V_{1}-V_{2}$ edges. Let $x=A e^{-c m} n$, where $A$ and $c$ are the constants obtained from Theorem 3.2. Then either the global condition $e\left(G_{2} \backslash G_{1}\right) \leq x$ fails, or $G_{2}$ has at most $x V_{1}-V_{2}$ edges. The former event has probability $o(1)$ by Theorem 3.2. To bound the probability of the latter event, we bound the probability $p$ that $G_{2}$, a standard random graph, contains any set $V$ of the appropriate size with a partition into parts $V_{1}, V_{2}$ of the appropriate sizes spanning at most $x$ edges. There are at most $\left(\begin{array}{c}n \\ |V|\end{array}\right)$ choices for the set $V$, and at most $2^{|V|}$ choices for the partition into $V_{1}, V_{2}$. Given $V_{1}$ and $V_{2}$, the number of $V_{1}-V_{2}$ edges in $G_{2}$ has a binomial distribution with mean $\mu=\left|V_{1}\right|\left|V_{2}\right| \eta m / n \geq 24 \eta(\log m)^{2} n / m$. Now $x<\mu / 100$ if $m$ is large enough, which we can enforce by the choice of $m_{0}$. Then, from the Chernoff bounds, the probability that given sets $V_{1}$ and $V_{2}$ span fewer than $x$ edges is at most $e^{-11 \mu / 12}$. Thus,

$$
p \leq\left(\begin{array}{c}
n \\
|V|
\end{array}\right) 2^{|V|} e^{-22 \eta(\log m)^{2} n / m}
$$


Using the bound $\left(\begin{array}{l}a \\ b\end{array}\right) \leq(e a / b)^{b}$, we have

$$
p \leq\left(\frac{2 e m}{10 \log m}\right)^{10 n \log m / m} \exp \left(-22 \eta(\log m)^{2} n / m\right) .
$$

Thus, for $m$ large enough,

$$
p \leq m^{10 n \log m / m} \exp \left(-22 \eta(\log m)^{2} n / m\right) .
$$

Since $22 \eta>10$ this is $o(1)$, completing the proof.

We now turn to coupling in the other direction. This time we give no explicit constants, as we shall be very cavalier with the constants in the proof.

Proof of Theorem 3.3. Let $\epsilon>0$ be given, and let $A$ be a large constant to be chosen later. We proceed as in the proof of Theorem 3.2, inductively constructing $G_{1}[k]$ and $G_{2}[k]$. This time we start at $k_{0}=\epsilon n$. Let us say that a vertex $i, 1 \leq i \leq k$, is bad at stage $k$ if $i$ has degree at least $A m$ in the graph $G_{1}[k]$. Note that if $i$ is bad at some stage, it is bad at all later stages, in particular, in the final graph $G_{1}$. From known results about the distributions of individual degrees in $G_{m}^{(n)}$ (see [Bollobás et al. 01]), whp the graph $G_{1} \sim G_{m}^{(n)}$ has at most $\epsilon n / m$ bad vertices with index $i \geq \epsilon n$ : for any vertex $i \geq \epsilon n$ and any $d \geq 0$, the probability that vertex $i$ has degree $d+m$ in $G_{m}^{(n)}$ is

$$
o\left(n^{-1}\right)+(1+o(1))\left(\begin{array}{c}
d+m-1 \\
m-1
\end{array}\right) \kappa^{m / 2}(1-\sqrt{\kappa})^{d},
$$

where $\kappa=i / n$. (See page 286 of [Bollobás et al. 01].)

As $\kappa$ is bounded away from zero, this is exponentially small in $d$ as $d \rightarrow \infty$. It follows that, given any $B>0$, if we choose $A$ large enough, then the expected number of bad vertices $i$ with $i \geq \epsilon n$ is at most $e^{-B m} n$. In particular, for a suitable choice of $A$, whp there are at most $\epsilon n / m$ such bad vertices.

Suppose that the graphs $G_{1}[k]$ and $G_{2}[k]$ are given, for some $k_{0} \leq k<n$. Let $t_{1}, \ldots, t_{m}$ be defined as in the proof of Theorem 3.2. Let $V_{k}$ be the set of vertices $j, k_{0} \leq j \leq k$, which are good, i.e., not bad, at stage $k$. Then for $1 \leq i \leq m$, conditional on $t_{1}, \ldots, t_{i-1}$, for each $j \in V_{k}$, we have

$$
\operatorname{Pr}\left(t_{i}=j\right) \leq(A m+m) /(2 m k) \leq A / k \leq A \epsilon^{-1} / n .
$$

Also, since the vertices before $k_{0}=\epsilon n$ all have degree at least $m$, and none of these is in $V_{k}$,

$$
\operatorname{Pr}\left(t_{i} \notin V_{k}\right) \geq k_{0} m /(2 m n)=\epsilon / 2 .
$$


Enumerate the vertices of $V_{k}$ as $j_{1}<j_{2}<\cdots<j_{k^{\prime}}$. For a fixed $i$ and for any $r$, given that $t_{i} \notin\left\{j_{1}, \ldots, j_{r-1}\right\}$, the probability that $t_{i}=j_{r}$ is at most $2 A \epsilon^{-2} / n$; here we use (4.2) to bound the numerator in the conditional probability, and (4.3) to bound the denominator below. It follows that we can construct a random subset $T_{i}$ of $V_{k}$, formed by selecting vertices of $V_{k}$ independently with probability $p=2 A \epsilon^{-2} / n$, in such a way that $t_{i} \in T_{i}$ whenever $t_{i} \in V_{k}$. In fact, as the relevant bounds hold conditional on $t_{1}, \ldots, t_{i-1}$, we can construct the sets $T_{i}$ so as to be independent of each other.

Putting the above together, let $S_{1}$ be the set of vertices in $V_{k}$ which are neighbours of $k+1$ in $G_{1}$. Then we can construct a random subset $S_{2}=T_{1} \cup$ $\cdots \cup T_{m}$ of $V_{k}$ so that $S_{1} \subset S_{2}$, and so that $S_{2}$ is obtained by selecting vertices in $V_{k}$ independently with probability $1-(1-p)^{m} \leq m p$. If we take $C$ large enough, then $C m / n \geq m p$, so we may construct $G_{2}[k+1]$ so that every vertex of $S_{2}$, and hence of $S_{1}$, is a neighbour of $k+1$ in $G_{2}$.

At the end of the construction we have coupled $G_{1}$ and $G_{2}$ so that, apart from multiple edges, an edge of $G_{1}$ not contained in $G_{2}$ is either incident with a vertex $i \leq \epsilon n$, or with a vertex $i \geq \epsilon n$ that was bad at some stage, and hence is bad in the final graph. As noted above, whp at most $\epsilon n / m$ vertices with index larger than $\epsilon n$ are bad in the final graph, while it is easy to check that whp $o(n / m)$ vertices are incident with multiple edges, completing the proof.

Part (b) of Theorem 3.1 now follows easily.

Proof of Theorem 3.I(b). Let $m_{0}$ be a large constant to be chosen later. Let us construct $G_{1}$ and $G_{2}$ coupled as in the statement of Theorem 3.3, taking $\epsilon=1 / 2$, and assuming (as we may) that the constant $C$ is greater than 2. It is well known that, if $a>1$ is a constant and $n \rightarrow \infty$, then whp the standard random graph $G(n, a / n)$ has independence number $(f(a)+o(1)) n$, where $f(a) \sim \log a / a$ as $a \rightarrow \infty$. (See, for example, the much more detailed result of Frieze [Frieze 90].) We shall apply this result to the subgraph of $G_{2}$ induced by the vertices with index larger than $n / 2$ : since this graph has the distribution of $G(n / 2, C m / n)=$ $G(n / 2,(C m / 2) /(n / 2))$, whp it contains an independent set of size at least (1$o(1)) f(C m / 2) n / 2$, which is asymptotically at least $n(\log m) /(C m)$. From the coupling given by Theorem 3.3, the graph $G_{1} \sim G_{m}^{(n)}$ contains an independent set of size at least $n(\log m) /(C m)-n /(2 m)$. If $c$ is any constant smaller than $1 / C$, and if $m$ is large enough, which can be enforced by the choice of $m_{0}$, then the final bound is at least $c n \log m / m$, completing the proof.

\section{Acknowledgments}

Research of the first author was supported by NSF grant ITR 0225610 and DARPA grant F33615-01-C-1900. 


\section{References}

[Albert et al. 00] R. Albert, H. Jeong, and A. -L. Barabási. "Error and Attack Tolerance of Complex Networks." Nature 406 (2000), 378-382.

[Albert and Barabási 02] R. Albert and A. -L. Barabási. "Statistical Mechanics of Complex Networks." Rev. Mod. Phys. 74 (2002), 47-97.

[Barabási and Albert 99] A. -L. Barabási and R. Albert. "Emergence of Scaling in Random Networks." Science 286 (1999), 509-512.

[Bollobás and Riordan 02] B. Bollobás and O. Riordan. "Mathematical Results on Scale-Free Random Graphs." In Handbook of Graphs and Networks, edited by Stefan Bornholdt and Heinz Georg Schuster, pp. 1-34. Weinheim, Germany: WileyVCH, 2002.

[Bollobás and Riordan 03] B. Bollobás and O. Riordan. "Robustness and Vulnerability of Scale-Free Random Graphs." Internet Mathematics 1 (2003), 1-35.

[Bollobás and Riordan 04] B. Bollobás and O. Riordan. "The Diameter of a ScaleFree Random Graph." To appear in Combinatorica. Preprint available from http://www.dpmms.cam.ac.uk/ omr10/.

[Bollobás et al. 01] B. Bollobás, O. Riordan, J. Spencer, and G. Tusnády. "The Degree Sequence of a Scale-Free Random Graph Process." Random Structures and Algorithms 18 (2001), 279-290.

[Buckley and Osthus 04] P. G. Buckley, and D. Osthus. "Popularity Based Random Graph Models Leading to a Scale-Free Degree Sequence." Preprint available from http://www.informatik.hu-berlin.de/ osthus/. To appear in Discrete Mathematics.

[Callaway et al. 00] D. S. Callaway, M. E. J. Newman, S. H. Strogatz, and D. J. Watts. "Network Robustness and Fragility: Percolation on Random Graphs." Phys. Rev. Lett. 85 (2000), 5468-5471.

[Cohen et al. 00] R. Cohen, K. Erez, D. ben-Avraham, and S. Havlin. "Resilience of the Internet to Random Breakdowns." Phys. Rev. Lett. 85 (2000), 4626-4628.

[Cohen et al. 01] R. Cohen, K. Erez, D. ben-Avraham, and S. Havlin. "Breakdown of the Internet Under Intentional Attack." Phys. Rev. Lett. 86 (2001), 3682-3685.

[Drinea et al. 01] E. Drinea, M. Enachescu, and M. Mitzenmacher. "Variations on Random Graph Models for the Web." Technical Report, Harvard University, Department of Computer Science, 2001.

[Dorogovtsev et al. 00] S. N. Dorogovtsev, J. F. F. Mendes, and A. N. Samukhin. "Structure of Growing Networks with Preferential Linking." Phys. Rev. Lett. 85 (2000), 4633.

[Dorogovtsev and Mendes 02] S. N. Dorogovtsev and J. F. F. Mendes. "Evolution of Networks." Adv. Phys. 51 (2002), 1079.

[Erdős and Rényi 59] P. Erdős and A. Rényi. "On Random Graphs I." Publicationes Mathematicae Debrecen 5 (1959), 290-297. 
[Frieze 90] A. Frieze. "On the Independence Number of Random Graphs." Discrete Math. 81 (1990), 171-175.

[Gilbert 59] E. N. Gilbert. "Random Graphs." Annals of Mathematical Statistics 30 (1959), 1141-1144.

Béla Bollobás, Department of Mathematical Sciences, University of Memphis, Memphis TN 38152, USA (bollobas@msci.memphis.edu)

Oliver Riordan, Royal Society Research Fellow, Department of Pure Mathematics and Mathematical Statistics, University of Cambridge, Cambridge, CB3 OWB, UK (o.m.riordan@dpmms.cam.ac.uk)

Received April 23, 2003; accepted July 25, 2003. 